\title{
Enhanced Cloud based Model for Healthcare Delivery Organizations in Developing Countries
}

\author{
Samuel, O.W. \\ Department of Computer \\ Science \\ Federal University of \\ Technology, Akure, \\ Nigeria
}

\author{
Omisore, M.O. \\ Department of Computer \\ Science \\ Federal University of \\ Technology, Akure, \\ Nigeria
}

\author{
Ojokoh, B.A. \\ Department of Computer \\ Science \\ Federal University of \\ Technology, Akure, \\ Nigeria
}

\author{
Atajeromavwo, E.J. \\ Department of Computer \\ Science \\ Delta State Polytechnic, \\ Ogwashi-uku \\ Ogwashi-uku, Nigeria
}

\begin{abstract}
Healthcare Delivery Organizations (HDOs) in developing countries have been faced with a number of problems due to their mode of operations. Amongst the problems are improper modes of data storage, insecurity of patient medical records, difficulty in accessing quality healthcare services, high cost of medical services, and inaccurate diagnosis and therapy procedures. In recent times, research has identified Cloud Computing (CC) as a new and substantial business model capable of providing efficient services that would benefit the healthcare industry. Despite its numerous advantages, $\mathrm{CC}$ has not been able to provide efficient means of securing medical data and it also lacks adequate data mining tools. This research therefore, proposes an enhanced model that integrates HDOs in developing countries into the cloud. The proposed model consists of a Data Security and User Authentication Engine (DSUAE) which prevents unauthorized access to patient medical records and as well employs standard encryption/decryption techniques to guarantee confidentiality of such records. The model also contains a Data Mining/Analysis and Pattern Prediction Engine (DMAPPE) which provides useful information that aids decision making through standard Data Mining techniques.
\end{abstract}

\section{Keywords}

Cloud Computing; Healthcare Organization; Data Mining; Data Security; Electronic Health Record.

\section{INTRODUCTION}

Cloud Computing (CC) is a general term for anything that involves the delivery of technological services over the Internet [1]. It is a model for enabling convenient and ondemand network access to a shared pool of configurable computing resources (for example, networks, systems, applications, and services) that can be rapidly provisioned and released with minimal management effort or service provider interaction [2]. CC and its business models have been some of the biggest changes impacting not only the computer industry but also several others in the twenty first century [3]. CC offers new and flexible ways to provision, manage, and pay for Information Technology (IT) resources. It paves way for new and more efficient business models and guarantees the achievement of organizational goals if properly exploited [2].

The Cloud has a number of infrastructure and multiple operating systems which enable consumers to build, test, and deploy their applications on virtual servers. In addition, the Cloud provides highly scalable environment for effective and efficient load handling [3]. It is characterized by: on-demand self-service; ever-present network access to computing resources; rapid and elastic provisioning with minimal management effort; and a pay-per-use scheme [2].

Healthcare Delivery Organizations (HDOs) in Nigeria and other developing countries have been faced with quite a number of challenges which have resulted to a high number death and morbidity in the region. In order to combat these challenges, some measures need to taken. Some of these measures, such as providing accurate medical diagnosis and improving health status of patients are embedded in the research work. Accurate medical diagnoses often aid therapy administration and as well improve the health status of patients [4]. Improved health status leads to increased productivity, educational performance, life expectancy, savings and investments, and decreased debts and expenditure on healthcare [5]. Ultimately, efficient healthcare delivery systems would lead to greater equity, economic return, and social/political stability in developing countries [6].

This research proposes a cloud based model with enhanced features that integrates HDOs in developing countries into the Cloud. The model is aimed at providing a platform that will adequately aid the operations of HDOs in developing countries by securing medical records and providing information that will assist various stakeholders and policy makers in decision making within the region.

The remaining part of this paper is structured as follows: Section 2 presents review of literature on $\mathrm{CC}$, challenges of HDOs in developing countries, and the benefits of CC; Section 3 presents the proposed model and methods adopted; Section 4 presents the conclusion which is drawn from the findings.

\section{RESEARCH BACKGROUND}

This section presents a review of research works that have been done concerning cloud computing; challenges faced by HDOs in developing countries; and the need for cloud computing in HDOs in the developing countries.

\subsection{Services and Deployment Models}

$\mathrm{CC}$ is an emerging computing paradigm that provides facilities needed to access shared resources and common infrastructure, offering services on demand over the Internet to perform operations that meet changing business needs of an organization [1]. CC is a new IT approach that offers new economic benefits, rapid deployment of services, and tight IT alignment with business goals [2]. It is not a new technology, but rather a new model for delivering computing resources and services in a more efficient and effective manner [7]. 
When CC is compared with conventional computing approach, it provides three key advantages which are: massive computing resources available on demand, elimination of an up-front commitment by users, and payment for use on a short term basis as needed by the consumer [8]. CC allows enterprises to get their applications up and running faster, with improved manageability and less maintenance, and enables IT to rapidly adjust resources to meet fluctuating and unpredictable business demands [9][10]. The applications of CC in industry, business, transportation, education, and national security have been reported in [11] [12] [13] [14].

Cloud Service Providers (CSPs) offer their services according to three fundamental models: Software as a Service (SaaS), Platform as a Service (PaaS), and Infrastructure as a Service (IaaS) [15][16]. In 2012, Network as a Service (NaaS) and Communication as a Service (CaaS) were officially included by the International Telecommunication Union (ITU) [17]. According to the United State (US) National Institute of Standards and Technology (NIST), there are four ways (models) of deploying CC: these are: Private Cloud, Public Cloud, Community Cloud, and Hybrid Cloud [18] [19].

\subsection{Challenges of HDOs in Developing \\ Countries}

Healthcare organizations constantly deal with large amount of electronic records and digital images from which useful knowledge about diagnosis of diseases, therapy administration, and managerial decisions can be drawn [20]. These records are often not properly kept in most developing countries either due to the use of inadequate traditional methods or lack of professionalism on the side of healthcare workers or lack of fund to adopt and adapt contemporary technological tools and devices [21]. As a result of the flaws associated with the orthodox approaches to medical services, many lives have been lost while several others have experienced serious deterioration in their health status [22].

In developing countries, much of the medical knowledge and information remains in paper form, and even when it is digitized, it often resides in disparate datasets and repositories and in diverse formats. Data sharing is uncommon and frequently hampered by the lack of foolproof de-identification for patient privacy. All these issues impede opportunities for data mining and analysis that would enable better predictive and preventive medicine [23].

Inadequate access to quality healthcare services constitutes a major challenge faced by HDOs in developing countries. This, no doubt, has contributed significantly to the ever growing death rate and it has equally resulted to reduced life expectancy in the regions involved. Those dwelling in rural communities of the developing countries usually find it extremely difficult to access quality healthcare services and infrastructure. This is because the few available healthcare infrastructures are located in urban centers [24].

Global shortage of adequately trained healthcare workforce is pervasive and impacts affordability and accessibility of quality healthcare delivery throughout the world. This problem is even worse in HDOs found in the developing countries [25]. As a result of this inadequacy, the cost of healthcare delivery becomes extremely high, while access to quality healthcare services becomes difficult [26].

\subsection{CC and HDOs in Developing Countries}

Healthcare organizations are expected to provide new and improved patient care capabilities at a reduced cost. IT plays a strong role in the health and patient care arenas with CC gradually beginning to make its marks [27]. Compared to other industries, the healthcare industry has significantly under-utilized IT to improve its operational efficiency.

Most HDOs in the developing countries still rely on paper medical records. Non-digitized information is often not portable and therefore inhibits its sharing amongst various healthcare delivery actors (patients, physicians, pharmacist, clinics, etc.). The use of technology to facilitate collaboration and coordinate healthcare between patients and physicians and amongst the medical community is still limited in Nigeria and other developing countries. The healthcare industry is shifting towards an information-centric care delivery model, enabled in part by open standards that support cooperation, collaborative workflows, and information sharing.

$\mathrm{CC}$ provides an enabling environment that allows hospitals, medical practices, insurance companies, and research facilities to tap improved computing resources at lower initial capital outlay. CC also provides a platform that reduces the barriers for innovation, modernization of Healthcare Information Technology (HIT) systems, and other healthcare related applications.

$\mathrm{CC}$ provides facilities that support big data sets for Electronic Health Records (EHRs), radiology images and genomic data offloading [28]. It also facilitates the sharing of EHRs among authorized physicians and hospitals in various geographic areas, providing more timely access to life-saving information there by reducing the need for duplicate testing [29]. Applications, services, and data can be accessed through a wide range of connected devices (e.g., smart phones, laptops, and other mobile internet devices).

HDOs data have stringent requirements for security, confidentiality, availability to authorized users, traceability of data, and long-term preservation. Hence, cloud vendors need to account for all these while conforming to government and industry regulations concerning healthcare records. 


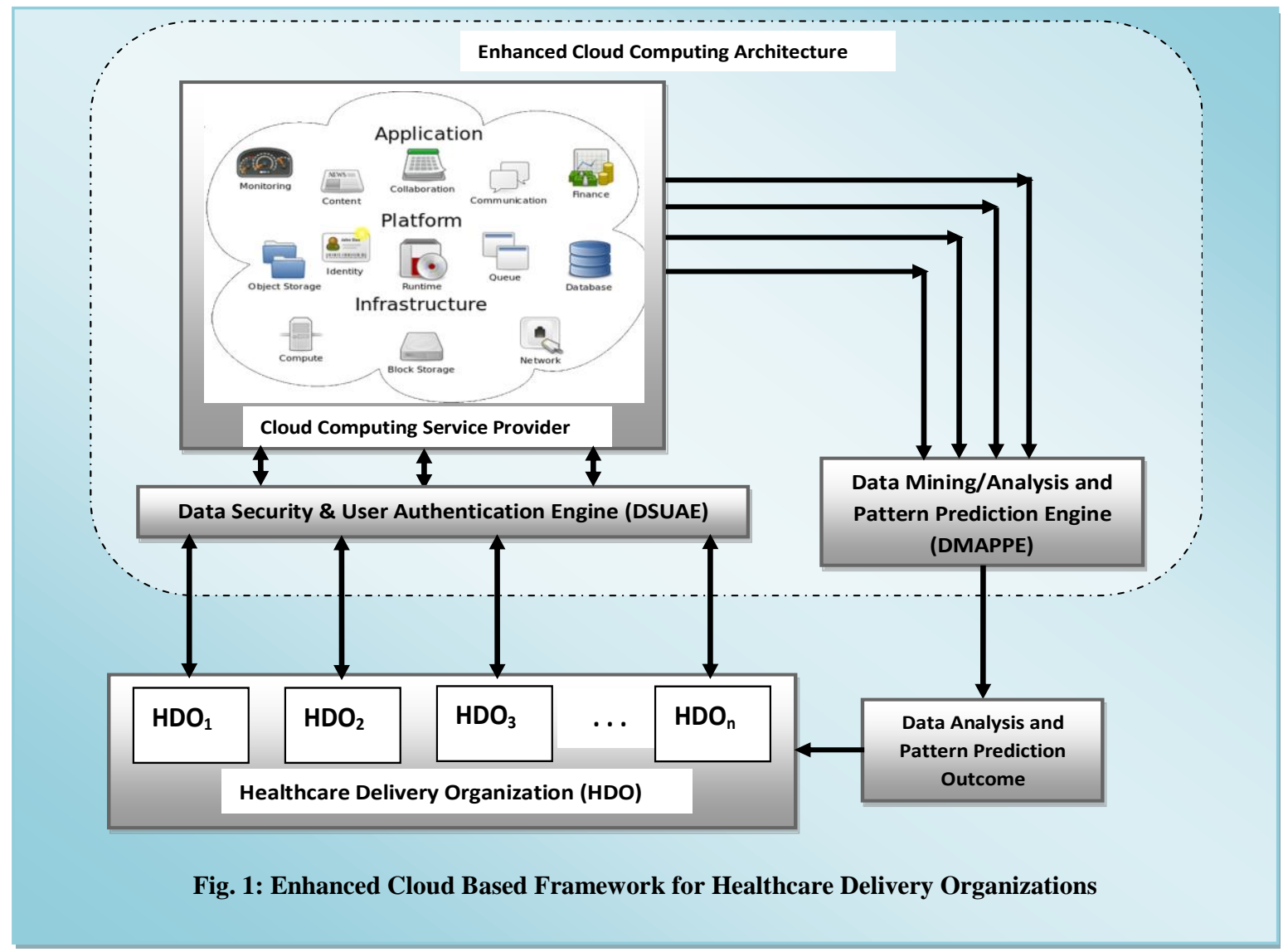

Despite the significant advantages offered by CC, security and privacy of medical data are some of the key challenges and barriers that have lead to its slow adoption by HDOs both in the developing countries and other parts of the world. Another major challenge hindering the adoption of $\mathrm{CC}$ services by HDOs is the lack of adequate Data Mining and Knowledge Discovery (DMKD) techniques. These DMKD techniques are capable of providing relevant information that could aid decision making in the healthcare industry.

\section{ENHANCED MODEL AND METHODS}

The architecture of the proposed model that integrates the cloud and HDOs in developing countries is presented in Figure 1. The architecture consists of a Cloud Service Provider (CSP), healthcare delivery organizations (HDOs), Data Security and User Authentication Engine (DSUAE) which protects medical records and prevents unauthorized access, Data Mining/Analysis and Pattern Prediction Engine (DMAPPE) which generates output that is capable of facilitating decision making in the health industry.

\subsection{HDOs and Cloud Service Models}

Table 1 presents the matrix of three CSPs and the basic cloud computing service models.

For instance, from Table 1, the categories of services offered by the cloud service provider with the code CSP1 is given by equation (1).

$$
\operatorname{CSP}_{1}=\left\{\mathrm{X}_{\operatorname{CSP}_{1}}, \mathrm{Y}_{\operatorname{CSP}_{1}}, \mathrm{Z}_{\mathrm{CSP}_{1}}\right\}
$$

Table 1. Cloud Service Provider versus Cloud Services

\begin{tabular}{|l|l|l|c|}
\hline & SaaS (X) & IaaS(Y) & PaaS(Z) \\
\hline $\mathbf{C S P}_{1}$ & $\mathrm{X}_{\mathrm{CSP}_{2}}$ & $\mathrm{Y}_{\mathrm{CSP}_{1}}$ & $\mathrm{Z}_{\mathrm{CSP}_{1}}$ \\
\hline $\mathbf{C S P}_{2}$ & $\mathrm{X}_{\mathrm{CSP}_{x}}$ & $\mathrm{Y}_{\mathrm{CSP}_{1}}$ & $\mathrm{Z}_{\mathrm{CSP}_{2}}$ \\
\hline $\mathbf{C S P}_{3}$ & $\mathrm{X}_{\mathrm{CSP}_{x}}$ & $\mathrm{Y}_{\mathrm{CSP}_{1}}$ & $\mathrm{Z}_{\mathrm{CSP}_{1}}$ \\
\hline
\end{tabular}

Each category of the cloud service model as in Table 1 contains several services. For example, some of the services contained in $\mathrm{X}_{\mathrm{CSP}_{1}}, \mathrm{Y}_{\mathrm{CSP}_{1}}$, and $\mathrm{Z}_{\mathrm{CSP}_{1}}$ are shown in equations (2), (3), and (4).

$\mathrm{X}_{\mathrm{CSP}_{1}}=\{\mathrm{EHR}, \mathrm{CRM}$, Email, Virtual Desktop, $\ldots$,

$$
\text { Google Applications }
$$

$\mathrm{Y}_{\mathrm{CSP}_{1}}=\{$ Virtual Machines, Servers, Storage,

$$
\begin{aligned}
& \text { Load balancers, ..., Network }\} \\
\mathrm{Z}_{\mathrm{CSP}_{1}=} & \{\text { Execution Runtime, Database }, \\
& \text { Web Servers, ..., Deployment Tools }\}
\end{aligned}
$$

Assuming HDO1, HDO2, and HDO3 subscribed for the EHR application, all CSPs that offer such a service must provide a general (standard) format for capturing medical data via the EHR application as shown by equation (5). 
Format $\left(\operatorname{EHR}\left[\mathrm{X}_{\mathrm{CSP}_{1}}\right]\right)=$ Format $\left(\mathrm{EHR}\left[\mathrm{X}_{\mathrm{CSP}_{2}}\right]\right)=$ Format

$$
\left.\left(\mathrm{EHR}\left[\mathrm{X}_{\mathrm{CSP}_{\mathrm{g}}}\right]\right)\right)
$$

This is necessary in order to ensure that all HDOs subscribing to the service of EHR supply medical data in the same format which would in turn facilitate efficient mining and analysis of all stored medical data and as well aid the discovery of hidden patterns that are necessary for effective decision making.

\subsection{Data Security and User Authentication Engine}

This component of the proposed model is powered by the Rivest Sharmir Adleman (RSA) cryptographic algorithm. This algorithm has been shown to be efficient in securing data, and it involves three key steps: key generation, encryption, and decryption. These steps are explained as follows:

- Key Generation: RSA algorithm makes use of public and private keys to encrypt and decrypt given messages respectively. Suppose $\mu=\{p, q, r, \ldots, v\}$ is a set of prime integers, such that any two elements (p and q) of $\mu$ with similar bit length are selected, then

$$
\mathrm{n}=\mathrm{pq}
$$

where $\mathrm{n}$ denotes the modulus of the public and private keys, while the length of $\mathrm{n}$ in bits denotes the key length. The Euler's Totient Function $(\varphi)$ of $n$ is computed by equation (7).

$$
\varphi(\mathrm{n})=(\mathrm{p}-1)(\mathrm{q}-1)
$$

Then an integer $e$ is chosen such that;

$$
1<\mathrm{e}<\varphi(\mathrm{n}) \text { and } \operatorname{gcd}(\mathrm{e}, \varphi(\mathrm{n}))=1
$$

That is, $e$ and $\varphi(n)$ must be co prime and $e$ is presented as the public key exponent. Lastly for the keys generation stage, the multiplicative inverse of e (d) is computed by equation (9) and $d$ is kept as the private key.

$$
d=e^{-1}(\bmod \phi(n))
$$

The public key consists of the modulus $\mathrm{n}$ and encryption exponent $\mathrm{e}$ while the private key consists of the modulus $\mathrm{n}$ and the decryption exponent $\mathrm{d}$, which must be kept secret. $p, q$, and $\varphi(n)$ must also be kept secret since they can be used to calculate $d$.

- Message Encryption: For instance, A sends his public key $(n, e)$ to B and keeps his private key secret. Then B sends a message (M) to A, by converting $\mathrm{M}$ into an integer $\mathrm{m}$, such that $0 \leq \mathrm{m}<\mathrm{n}$, by using an agreed upon reversible protocol known as a padding scheme. B then proceeds to compute the cipher text (C) of $\mathrm{m}$ by using equation (10) before sending it to A.

$$
\mathrm{C} \equiv \mathrm{m}^{\mathrm{e}}(\bmod \mathrm{n})
$$

\section{CONCLUSION}

A significant number of death is been recorded yearly due to the way HDOs in developing countries operates. Some of the factors responsible for this are; high cost of medical services, inadequate access to quality healthcare personnel and infrastructure, inaccurate diagnostic and therapy procedures, and poor storage of medical/clinical data. This research
- Decrypting the Cipher Text: A can now recover m from C by using his private key exponent (d) via equation (11). To recover the original message $\mathrm{M}$ form $\mathrm{m}$, $\mathrm{A}$ will have to reverse the padding process that was earlier initiated by $\mathrm{B}$

$$
m \equiv C^{\mathrm{e}}(\bmod \mathrm{n})
$$

\subsection{Data Mining/Analysis Pattern Prediction Engine}

This component uses k-nearest neighbor $(\mathrm{kNN})$ classification algorithm whose efficiency is proven by [30]. kNN algorithm finds a group of $\mathrm{k}$ objects in a given training set that are closest to a test object (object under consideration), and places the assignment of a label on the predominance of a particular class in the neighborhood. This technique is made up of three steps: a set of labeled objects, e.g., a set of stored records; a distance or similarity metric to compute the distance between objects; and the value of $\mathrm{k}$ (the nearest neighbors).

To classify an unlabeled object, the distance of the object to the labeled objects is computed, its kNNs are identified, and the class labels of these nearest neighbors are then used to determine the class label of the object. The kNN classification method in presented as follows:

Given a training set $\mathrm{D}$ and a test object $\mathrm{x}=\left(x^{f}, y^{y}\right)$, the Euclidean Distance (ED) formula used to compute the distance (or similarity) between $\mathrm{z}$ and all the training objects $(\mathrm{x}, \mathrm{y}) \in \mathrm{D}$ to determine its nearest-neighbor list, $\mathrm{Dz}$ is presented by equation (12), where $\mathrm{x}$ is the data of a training object, while y is its class. Likewise, $x^{x}$ is the data of the test object and $y^{f}$ is its class.

$$
\mathrm{ED}(\mathrm{z}, \mathrm{x})=\sqrt{\left(z_{1}-x_{1}\right)^{2}+\left(z_{2}-x_{2}\right)^{2}+\cdots+\left(z_{n}-x_{n}\right)^{2}}
$$

for an n-dimensional space. Once the nearest-neighbor list is obtained, the test object is classified based on the majority class of its nearest neighbors by using equation (13).

$$
\text { Majority Voting }\left(y^{f}\right)=\operatorname{argmax} \sum_{(x i y i) \in D z}^{1} I(v=y i)
$$

where $\mathrm{v}$ is a class label, yi is the class label for the ith nearest neighbors, and $\mathrm{I}(\cdot)$ is an indicator function that returns the value 1 if its argument is true and 0 otherwise. A summary of the $\mathrm{kNN}$ classification algorithm is presented as follows:

Input $\rightarrow$

$\mathrm{D}$, the set of $\mathrm{k}$ training objects, and test object $\mathrm{z}=\left(\mathrm{x}^{f}, \mathrm{y}^{\prime}\right)$.

Process $\rightarrow$

Computed $\left(\mathrm{X}^{f}, \mathrm{x}\right)$, the distance between $\mathrm{z}$ and every object, (x, y) $\in$ D.

Select $\mathrm{Dz} \subseteq \mathrm{D}$, the set of $\mathrm{k}$ closest training objects to $\mathrm{z}$.

Output $\rightarrow$

$y^{\prime}=\operatorname{argmax} \sum_{(x i, y i) \in D z}^{1} \mathrm{I}(v=y i)$.

provides an Enhanced Cloud Computing Platform that is capable of integrating Healthcare Delivery Organizations in developing countries into the cloud in order to provide them with efficient mode of operation. The proposed platform incorporates a DSUAE that guarantees the security and confidentiality of patients' medical records as well as prevents unauthorized access to such records. The platform also 
provides a means via which useful information can be mined through its DMAPPE. Lastly, the information provided from the DMAPPE could aid effective decision making by the management of HDGs or other concerned stakeholders and as well lead to social/economic stability of nations of the developing countries.

However, healthcare organizations in the developed countries of the world can as well benefit tremendously from the enhanced cloud based platform proposed by this research. The platform can as well be adapted to suit the needs of business organizations outside the healthcare industry.

\section{REFERENCES}

[1] Srinivasa, R., Nageswara, R., and Ekusuma, K., 2009. “ Cloud computing: An overview," Journal of Theoretical and Applied Information Technology (JATIT), Pp. 7176.

[2] IBM Corporate Marketing White paper, "Cloud computing: Building a new foundation for Healthcare,". ibm.com/cloud, 2011.

[3] Sanjay, P. A., Sindhu, M., and Jesus, Z. 2012. "A Survey of the state of Cloud computing in Healthcare," in Canadian Center of Science and Education, Network and Communication Technologies; Vol. 1, No. 2; ISSN 1927-064X E.

[4] Samuel, O. W., Omisore, M. O., and A. Ojokoh, B. 2013. "A Web Based Decision Support System Driven by Fuzzy Logic for the Diagnosis of Typhoid Fever," in Expert Systems With Applications Expert Systems With Applications 40, pp. 4164-4171 DOI information: http://dx.doi.org/10.1016/j.eswa.2013.01.030

[5] Ibekwe, P. C., 2010 "Healthcare problems in Developing Countries," Medical Practice and Reviews, Vol. 1(1), Pp. 9-11.

[6] Dan, K. 2006. "Healthcare in Africa: Challenges, Opportunities and an Emerging Model for Improvement," Presented at the Woodrow Wilson International Center for Scholars, November 2, 2006.

[7] Kuo, A. M., 2011. "Opportunities and Challenges of Cloud computing to Improve Healthcare Services," Journal of Medical Internet Research (JMIR), Vol. 13 No.3, e67. http://.doi.org/10.2196/jmir.1867

[8] Armbrust, M. A., Fox, R., Griffith, A. D., Joseph, Katz, R., and Konwinsiki, A. 2010. "A view of Cloud computing," Communications of ACM, 53(4), Pp. 50-58.

[9] Baburajan, R., 2013. "The rising Cloud Storage Market Opportunity Strengthens Vendors," info TECH, August 24, 2011, http://it.tmcnet.com/channels/cloudstorage/articles/211183-rising-cloud-storage-marketopportunity-strengthens-vendors.htm. Date Accessed: 04-02-2013-04.

[10] Oestreich, K. 2013 "Converged Infrastructure," CTO Forum, November $\quad 15, \quad 2010$. http://www.thectoforum.com/content/convergedinfrastructure-0. Date Retrieved 2013-04-02.

[11] Technology Firms and Health Care, "Heads in the Clouds-Digitizing America's Health Records Cloud be a Huge Business. Will It?" The Economist (US)2011; 399(8727):63.

[12] Li, Z. J., Chen, C., and K. Wang, Cloud computing for Agent-Based Urban Transportation Systems, IEEE Intelligent System. 2011; 26(1):73-97.

[13] T.S. Behrend, E. N. Wiebe, J. E. London., and E.C. Johnson "Cloud computing Adoption and Usage in
Community Colleges," Bohave Information Technology 2011; 30(2):231-240.

[14] Dark Government, "NSA-Embraces Cloud computing. URL: $\quad$ http://www.darkgovernment.com/news/nsaembraces-cloud-computing, Date Retrieved: 02-012013.

[15] National Institute of Standards and Technology, "The Definition of Cloud computing," http://csrc.nist.gov/publications/nistpubs/800-145/SP800145.pdf. Date Retrieved 15 -01-2013.

[16] R. Buyya, J. Broberg, and A. Goscinski, "Cloud computing Principles and Paradigms," New York, USA, Wiley Press. pp. 1-44, 2011.

[17] International Telecommunication Union, "ITU News Log - Cloud Computing and Standardization: Technical Reports,".http://www.itu.int/ITUT/newslog/Cloud+Com puting+And+Standardization+Technical+Reports+Publis hed.aspx Date Retrieved 16-12-2012.

[18] P. Mell, and T. Grace, "The National Institute of Standard and Technology (NIST)," Definition of Cloud computing," Communications ACM 2010, 53(6):50.

[19] H. F. Cervone, "An Overview of Virtual and Cloud computing.," OCLC System Services 2010; 26(3): 162165.

X. Wang, "Application of Cloud computing Healthcare Information System," Computer Application and System Modeling,

2010. http://ieeexplore.ieee.org/stamp/stamp.jsp?tp=\&armnum ber=5619051Date Retrieved: 28-12-2012.

[20] L. Kohn, J. Corrigan, and M. Donaldson, "Eds. To Err Is Human: Building a Safer Hearlth System," Washington, D.C., National Academic Press, 2000.

[22] Y. K., Meng, "Interval-Based Reasoning in Medical Diagnosis," Proceedings of National Conference on Research and Development in Computer Science and Its Applications (REDECSA) 1996, Universiti Pertanian Malaysia: Kuala Lumpur, pp. 220 - 224.

[21] J. Roberson, and D. Dehart, "Robertson Research Institute" \& K. Toll, and D. Hecherman, "Microsotf Research,"' Healthcare Delivery in Deloping Countries: Challenges and Potential Solutions," 2009.

[22] D. Simba, K. Dan, and G. Kiangi, "Community Based Health Care: The case of Mufindi District, Tanzania," Tropical Institute of Community Health Publication, Nairobi, Kenya, 2003.

[23] CG Insights, "The Global Challenge of Healthcare Delivery," Vol. 1 No. 1, May 2008 Marguerite Moore Callaway.

[24] Human Resources in Africa, "Issues Paper", http://www.healthgap.org/camp/hcw_docs/USAID_healt hsector_africa.pdf. Date Accessed 17-12-2012

[25] IBM Research Collaborations with Leading Taiwanese Institutions to Deliver Wellness-Centric Healthcare via Cloud Computing, IBM Press Release, 2010.

[26] C. O. Rolin, et al. "A Cloud computing Solution for Patient's data collection in Healthcare Institutions," Proceedings of IEEE on the 2nd International Conference on eHealth, Telemedicine, and Social Medicine, February 10-16, 2010, New York, U.S.

[27] L. Guo, F. Chen, L. Chen, and X. Tang, "The Building of Cloud computing Environment for E-Health Networking, Digital Ecosystems and Technologies(EDT)," IEEE International Conference on E-health Networking, July, 1-3, 2010, Lyon, France.

[29] W. Xindong, et al, "Top 10 Algorithms in Data Mining," Knowledge Information System, Vol. 14, Pp. 1-37, 2008. DOI: 10.1007/s10115-007-0114-2. 


\section{AUTHORS PROFILE}

Mr. Samuel Oluwarotimi Williams has B.Sc. degree in Computer Science and he is currently pursuing a Maters of Technology Degree in Computer Science at the Federal University of Technology, Akure, Nigeria. He has over five years experience in teaching and research in the field of computing. He has special interest in Computational Intelligence, Cloud Computing, and Programming. He currently works with High Technology Research and Development Group (HTRDG) Computer LTD, Nigeria as a Research Assistant/Software Engineer.

Contact: +2348032397138, timitex92@gmail.com

Mr. Omisore Mumini Olatunji is a Research Student at the Federal University of Technology, Akure, Nigeria. He Studied Computer Science at Undergraduate Level and currently on his Masters of Technology Degree in Computer Science (Software Engineering and Database System). He has worked for over two years as Research Assistant/System Analyst at High Technology Research and Development Group (HTRDG) Computer Limited, Nigeria.

Contact: +2347031967847, ootsorewilly@gmail.com
Dr. (Mrs.) Bolanle Adefowoke Ojokoh has B.Sc, M.Tech and $\mathrm{Ph} . \mathrm{D}$. degrees in Computer Science. She has been involved in teaching Computer Science courses for over 10 years in the Federal University of Technology, Akure, Nigeria. Her research interests include metadata extraction, digital libraries, recommendation systems and gender issues in ICT. She has published several papers in learned journals and academic conferences. She was on Postgraduate and Postdoctoral Research visit to Peking University, China. Contact: +2347030538346, bolanleojokoh @yahoo.com

Mr. Atajeromavwo Edafe John has B.Tech and M.Tech. degree in Computer Science at the Federal University of Technology, Akure, Nigeria. He is currently pursuing a Ph.D in the same University. He currently works as a Principal Lecturer in the department of Computer Science Delta State University, Ogwashi-uku, Nigeria. His areas of research interests include Software Engineering, Mobile Computing, Distributed Computing, and Database Systems.

Contact: +2348064784094, edafejohn2006@yahoo.com 